\title{
Fístula de líquido cefalorraquídeo espontánea: meningocele de pared lateral de esfenoides
}

\section{Reporte de un caso}

Del Río Cardona Sergio Alejandro*, Andrade Lozano Paulina**, Salas Galicia Juan Eugenio***

Resumen

- Las fistulas de líquido cefalorraquídeo son el resultado de las - dehiscencias óseas y adelgazamientos parciales que pueden

- debilitar la barrera normal entre el espacio subaracnoideo y

- la cavidad sinonasal; éstas pueden llegar a ser tan grandes,

- que pueden llegar a favorecer la herniación de la duramadre

- y la aracnoides hacia la cavidad nasal, sitio de menor resis-

- tencia de la base del cráneo. Las fístulas de líquido cefalorra-

- quídeo (FLCR) espontáneas, por lo general, se establecen en

- el área de la lámina cribosa donde la duramadre alrededor

- del nervio olfatorio parece tener una extensión a través de

- ésta. Otro sitio común es a través de un seno esfenoidal muy

- neumatizado donde un sistema de alta presión puede ser

- un factor contribuyente. Se presenta el caso clínico de una

- paciente del sexo femenino de 49 años con antecedente

- de haber tenido un trauma craneoencefálico que presenta

- posteriormente FLCR. LUXMÉDICA AÑO 15, NÚMERO 44, MAYO-

AGOSTO 2020, PP 49-55.

Palabras clave: meningocele, fístula de líquido cefalorraquídeo, rinorrea, seno esfenoidal.

\section{Abstract}

Cerebrospinal fluid fistulas are the result of bone dehiscence and partial thinning that can weaken the normal barrier between the subarachnoid space and the sinonasal cavity. These can become so large that it can favor herniation of the dura and arachnoid into the nasal cavity, this site is the least resistant at the base of the skull. Spontaneous cerebrospinal fluid fistulas (CSFF) usually present in the cribriform plate where the dura around the olfactory nerve appears to extend through it. Another common site is through a highly pneumatic sphenoid sinus where a high-pressure system could be a contributing factor. We present a 49-year-old female patient with a history of head trauma, who subsequently presented a CSFF. LUXMÉDICA AÑO 15, NÚMERO 44, MAYO-AGOSTO 2020, PP 49-55.

Keywords: meningocele, cerebrospinal fluid fistula, rhinorrhea, sphenoid sinus

* Estudiante de séptimo semestre de la carrera de Médico Cirujano del Centro de Ciencias de la Salud de la Universidad Autónoma de Aguascalientes

** Profesor Investigador en la Universidad Autónoma de Aguascalientes. Médico adscrito al servicio de Otorrinolaringología, Centenario Hospital Miguel Hidalgo, Aguascalientes, Ags., México.

*** Otorrinolaringólogo y Cirujano de Cabeza y Cuello, Clínica Médica Santé, Veracruz, Ver, México.

Fecha de recibido: 18 de febrero 2020

Fecha de aceptación: 28 de abril 2020

Correspondencia Dra Paulina Andrade Lozano Departamento de Medicina, Edificio 107, planta alta. Centro de Ciencias de la Salud. Campus universitario. Universidad Autónoma de Aguascalientes. Avenida Universidad 940. Código postal 20131. Aguascalientes, Ags., México. Teléfono 449 9108443. Correo electrónico drapaulinaandrade15@gmail.com 


\section{Introducción}

El término de fístula de líquido cefalorraquídeo (FLCR) implica la comunicación con el espacio subaracnoideo a través de un rompimiento estructural en la base del cráneo. ${ }^{1}$ Por tanto, conllevan implícitamente una solución de continuidad de la duramadre que permita la comunicación entre el espacio subaracnoideo y las estructuras nasosinusales o el oído medio (es decir, ocurren debido a la existencia de un defecto osteodural). ${ }^{2}$ Se necesitan tres factores concomitantes para que se produzca una fístula de LCR: defecto óseo, disrupción meníngea y un gradiente de presión. ${ }^{3}$

Según su etiología, la clasificación de las fístulas craneosinusales se divide en adquiridas y espontáneas. Las fístulas traumáticas, que son una derivación de las adquiridas, llegan a ser hasta un $90 \%$ del total y generalmente son por fracturas localizadas en la base del cráneo. Los tres principales sitios de fractura se dividen en centrales (seno esfenoidal y selares), de fosa craneal anterior (lámina cribosa, seno frontal) y las laterales (sobre hueso temporal). En la misma clasificación, dentro de las traumáticas, se encuentran las iatrogenias ocasionadas por procedimientos neuroquirúrgicos o por cirugía endoscópica nasosinusal. Las fístulas de líquido cefalorraquídeo adquiridas no traumáticas se deben generalmente a tumores de la base del cráneo en donde tienden a tener un mecanismo de acción sobre el hueso adyacente, ya sea de manera directa (erosión ósea) e indirecta (hidrocefalia, secuela de quimioterapia o radioterapia). Dentro de las fístulas de tipo espontáneas que son el tema principal de este reporte de caso, se encuentran las congénitas y las idiopáticas. Las fístulas congénitas generalmente debutan durante la infancia; en cambio, las fístulas idiopáticas debutan en la edad adulta. ${ }^{4}$

Los pacientes con fístulas espontáneas presentan rinorrea acuosa por una o ambas fosas nasales, por lo que, con frecuencia, son tratados como rinitis y no tienen antecedentes traumáticos o quirúrgicos. Hay distintas teorías acerca de la etiología de las fístulas espontáneas; algunos autores proponen que las fístulas se producen a través de dehiscencias congénitas de la base del cráneo; sin embargo, se presta a discusión si estas dehiscencias son congénitas o son consecuencia de la remodelación o reabsorción ósea a lo largo de la vida del paciente..$^{2,5}$

Existen características específicas en los pacientes con fístulas espontáneas, y se han descrito con las 5 " $\mathrm{F}$ " female, fat, forty, five feet tall y fearfulface que caracterizan el fenotipo. ${ }^{6}$ Las pacientes obesas con un índice de masa corporal superior a 35 tienden a hacer maniobra de Valsalva constantemente por los esfuerzos que deben hacer al día a día, que conduce a un incremento de la presión intracraneal, provocando erosiones sobre la base del cráneo, lo cual favorece la creación de fístulas espontáneas. En ocasiones, estas erosiones y defectos osteodurales llegan a ser tan grandes que permiten la salida de estructuras de la cavidad craneal y for- 
man un meningocele, meningoencefalocele, hernia meníngea o aracnoidea. Estas diferentes acepciones reflejan la variabilidad del tejido herniado a través del defecto osteodural, y ocasionalmente la imprecisión para definirlos no sólo con técnicas de imagen sino también durante la cirugía, debido al tamaño del campo quirúrgico y de la propia lesión. 2,6

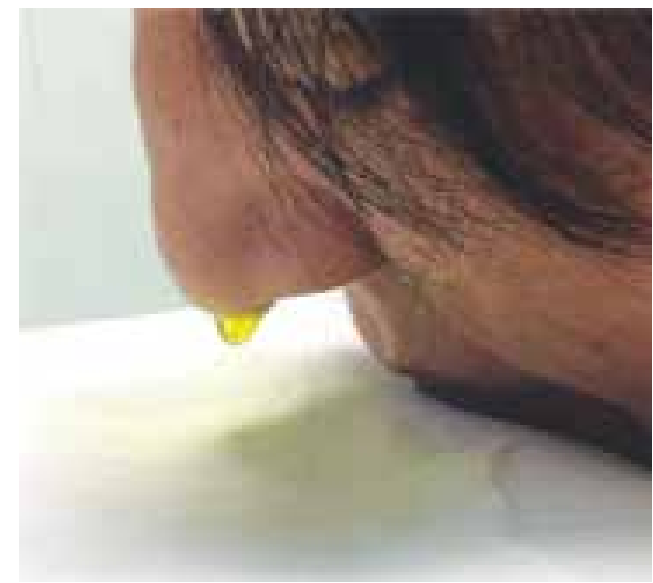

Figura 1. Rinorraquia o rinorrea de líquido cefalorraquídeo. En este caso se hizo tinción con fluoresceína intratecal para confirmación del diagnóstico.

\section{Presentación del caso clínico}

Paciente del sexo femenino de 49 años con antecedente de haber sufrido un trauma craneoencefálico hace cinco años sin complicaciones. Antecedentes alérgicos, médicos, quirúrgicos y transfusionales interrogados y negados. Inicia su padecimiento actual tres meses antes de acudir a consulta médica por rinorrea anterior hialina, acuosa, transparente y bilateral de predominio derecho, la cual aumenta al cargar cosas pesadas. Refiere además obstrucción nasal intermitente, hiposmia y cefalea frontal, la cual mejora con la administración de analgésicos. La paciente fue diagnosticada con rinitis alérgica y recibió tratamiento a base de antihistamínicos (loratadina) y esteroide nasal (mometasona), con mejoría parcial de la sintomatología obstructiva; sin embargo, continuó con rinorrea anterior hialina, la cual deglute -describe que es de sabor salado- e irrita la faringe, por lo que es canalizada a consulta con especialista en otorrinolaringología.

Fue sometida a una endoscopía nasal donde se encontraron los cornetes con mucosa normocrómica de aspecto polipoideo (cola de cornete medio e inferior), y salida de líquido transparente acuoso por receso esfenoetmoidal derecho, el cual aumentaba a la realización de la maniobra de Valsalva. Se realizó el estudio citoquímico de líquido de la rinorrea, el cual fue positivo a líquido cefalorraquídeo. Se realizó tomografía simple de nariz y senos paranasales donde se observó densidad de tejidos blandos que ocupa hemiseno esfenoidal derecho en su totalidad, así como solución de continuidad de pared lateral de seno esfenoidal. Por lo que se indicó la realización de resonancia magnética nuclear $(\mathrm{RMN})$ en la cual se observó una lesión hipointensa en T1 e hiperintensa en T2 que ocupaba el 100\% del seno esfenoidal. Al momento, se le diagnosticó meningocele esfenoidal derecho. 


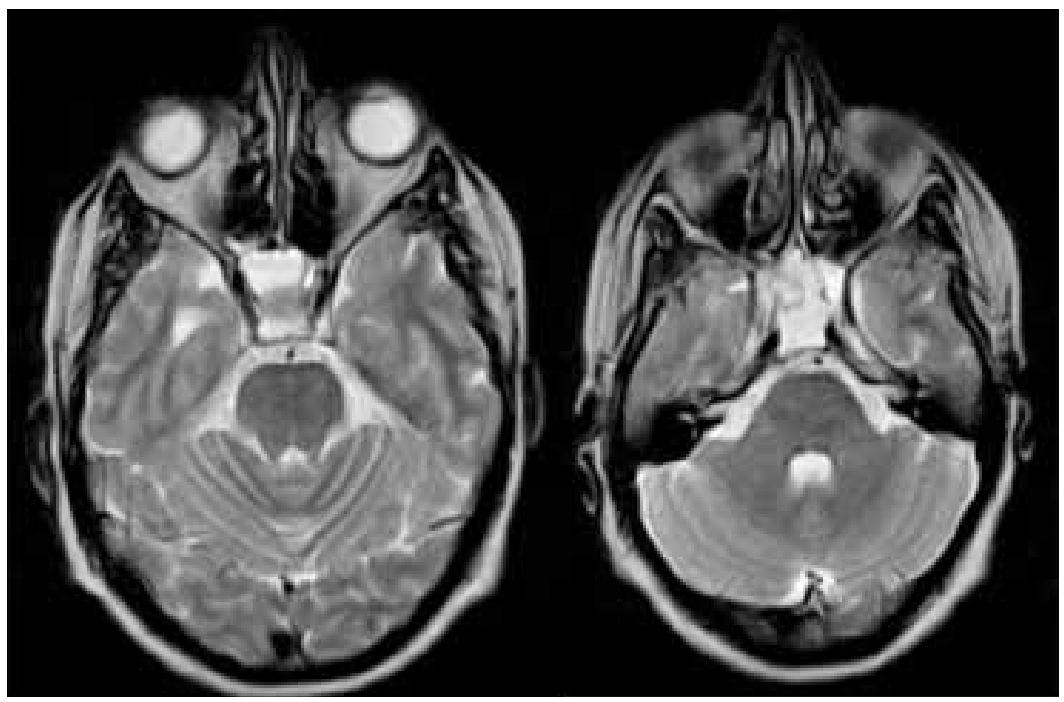

Figura 2. RM T2 ponderada donde se observa lesión hiperintensa que ocupa el 100\% del seno esfenoidal, datos sugestivos de meningocele. $R M$ resonancia magnética.

Se le realizó la resección endoscópica del meningocele con cierre del defecto en la pared lateral del esfenoides. Se llevó a cabo el cierre multicapa de defecto con injerto de cartílago grasa inlay (intracraneal), injerto libre de mucosa de cornete medio overlay (sobre el defecto) y, finalmente, se colocó un colgajo nasoseptal (Haddad); todos los injertos y colgajos estuvieron sumergidos en plasma rico en factores de crecimiento como pegamento autólogo, así como el colgajo nasoseptal de Haddad. Se mantuvo a la paciente con taponamiento anterior bilateral, reposo absoluto e inhibidores de la anhidrasa carbónica durante una semana. Se retiró el taponamiento anterior y se realizaron curaciones endoscópicas en consultorio durante tres semanas. Se obtuvo un cierre exitoso de la fístula en el primer intento quirúrgico. La paciente ha tenido seguimiento de un año.

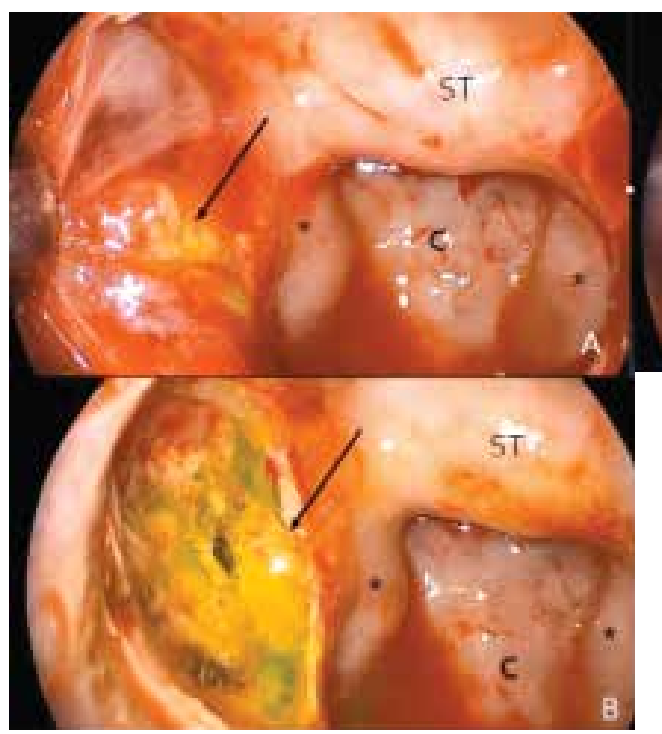

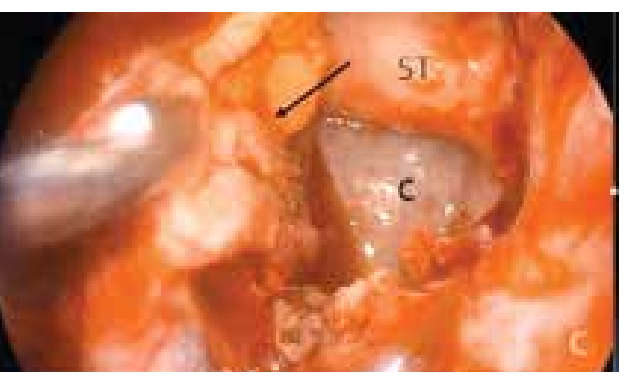

Figura 3. flecha: meningocele dependiente de la pared lateral del seno esfenoidal (derecho) B. flecha: posterior a la resección del meningocele se observa defecto óseo en pared lateral del seno esfenoidal. C. cierre del defecto óseo con técnica endoscópica multicapa con grasa, cartílago y mucosa. ST: silla turca, $C$ : clivus, *: protuberancia de la arteria carótida interna. 


\section{Discusión}

La relevancia de este caso se debe a que muchas veces las FLCR no son diagnosticadas oportunamente, provocándole al paciente un riesgo, ya que estas patologías son potencialmente mortales por las posibles complicaciones. La meningitis bacteriana es la principal causa de morbilidad y mortalidad en estos pacientes y a menudo es el primer signo clínico de este padecimiento. ${ }^{3}$

En 1940 se comunicó por primera vez el procedimiento de la reparación intracraneal de una FLCR a través de una craneotomía bifrontal. A partir de entonces, fueron surgiendo mejoras en el tratamiento quirúrgico, como el abordaje extracraneal a través de una incisión nasoorbitaria, o las técnicas con base en la visión microscópica, así como el cierre mediante endoscopía a través de una etmoidectomía. Se le debe a Messerklinger el uso de la fluoresceína intratecal para la localización exacta de las fístulas de LCR en la fosa craneal anterior. ${ }^{5}$

La rinorrea de líquido cefalorraquídeo (LCR) generalmente es el principal síntoma y resulta de la comunicación directa del espacio subaracnoideo y el espacio tapizado de mucosa de la nariz y los senos paranasales. El paso de líquido cefalorraquídeo hacia la cavidad nasal se define como rinorraquia o rinolicuorrea y St. Clair Thompson la describió en 1899 como una entidad patológica. ${ }^{7}$ Durante las décadas subsecuentes, se reconoció la importancia clínica de las fístulas. ${ }^{6}$ La lámina lateral de la placa cribiforme, que es más delgada que las porciones más laterales del techo del etmoides, ha sido descrito como el más susceptible al daño. En el $2 \%$ de todos los traumatismos de la cabeza, el 12\%-30\% de las fracturas de la base del cráneo y el $25 \%$ de las fracturas faciales, se ha notificado la presencia de rinorrea del LCR. ${ }^{8}$

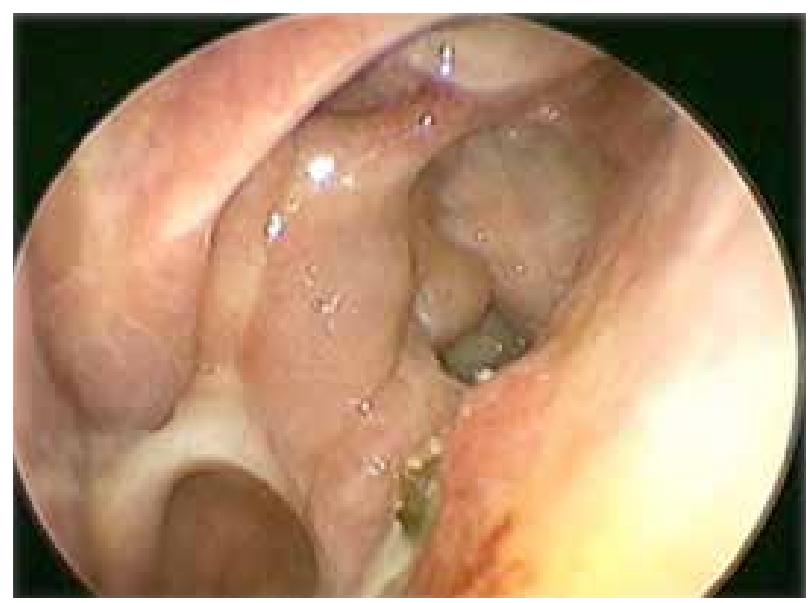

Figura 4. Evolución postquirúrgica, 10 meses después de la cirugía, sin datos de fístula y con función del seno esfenoidal normal.

El manejo de las FLCR ha cambiado en los últimos años, gracias a la introducción de la cirugía endoscópica, que permite abordajes mínimamente invasivos por endoscopía endonasal con abordaje extradural y ha demostrado ser una técnica segura y eficaz en el manejo de las fístulas de LCR disminuyendo el riesgo de morbilidad, el riesgo de alteración en el sentido del olfato y los días de hospitalización del paciente. ${ }^{3,4,8,9}$

La mayoría de las series apuntan a un $90 \%$ de éxito. El entendimiento de la fisiopatología, diagnóstico y tratamiento es 
imperioso para el tratamiento adecuado de esta condición. El uso de endoscopios para reparar las fístulas del líquido cefalorraquídeo tiene múltiples ventajas sobre otros abordajes, incluyendo mejor visión con mejor iluminación y ángulo de visión magnificado. Otras ventajas incluyen la habilidad para retirar la mucosa del hueso adyacente sin incrementar el tamaño del defecto y verificar el posicionamiento del injerto bajo visión directa. Esto requiere menor tiempo quirúrgico y está asociado a menor morbilidad. Esta técnica debe ser considerada como el método de primera elección para cierre en todos los casos de fístula, excepto en aquellos asociados con patología intracraneal obvia, como una lesión ocupante de espacio. ${ }^{11-13}$

Para el diagnóstico, es conocido que el estudio de laboratorio para analizar el líquido de rinorrea está relacionado con alta tasa de falsos positivos; además, la evaluación radiológica no es $100 \%$ espe- cífica para localizar el lugar de la fístula. El uso de fluoresceína intratecal es un excelente método diagnóstico del sitio de fístula de líquido cefalorraquídeo. ${ }^{7,9}$ Por ende, el diagnóstico de las fístulas de líquido cefalorraquídeo debe estar basado en una combinación de estudios de laboratorio y de radiología. Las FLCR espontáneas suponen un reto quirúrgico debido a sus altas tasas de recurrencia. Esto se puede atribuir a la alta prevalencia de hipertensión intracraneana en este grupo de pacientes. Es por eso que se debe añadir una fuerte estrategia de tratamiento para esta patología después del cierre endoscópico para disminuir la tasa de recurrencia. En los pacientes con FLCR de origen espontáneo, es importante el monitoreo postquirúrgico de la presión intracraneana así como el uso de inhibidores de la anhidrasa carbónica (acetazolamida) y un drenaje subaracnoideo, para disminuir la falla postquirúrgica. ${ }^{1,3}$

\section{Conclusiones}

Cualquier fístula de la base del cráneo puede ocasionar graves complicaciones, incluso aquellos defectos que son pequeños y de bajo gasto. Es por lo anterior que el médico general debe conocer la patología y las posibles complicaciones de las FLCR para que se haga el diagnóstico oportuno al paciente y reciba el tratamiento adecuado.

\section{Bibliografía}

1. González Sánchez Jorge Glicerio, Chávez Velázquez Ana Milagros, Velázquez Santana Héctor. Manejo integral de fístulas de líquido cefalorraquídeo. AN ORL MEX 2012;57(1):25-30.

2. Cano-Alonso $R$, Jiménez-De La Peña $M$, GómezCaicoya $A$, Recio-Rodríguez $M$, Álvarez-Moreno $E_{\text {, }}$ Herraiz-Hidalgo L, Pozuelo de Alarcón, Madrid. Fístulas espontáneas de la base del cráneo en el adulto: poco frecuentes, pero importantes. Sociedad Española de Radiología Médica. 2012; 73:1-31 10.1594/ seram2012/S-0073.

3. Robledo-Moreno Edgar, Vargas-Olguín Eduardo, González-Sánchez Jorge Glicerio, Bribiescas-Carreón
Lizeth. Fístula espontánea de líquido cefalorraquídeo a través del clivus. Arch Neurocien (Mex) INNN 2016;21(3):39-42. Disponible en https://www.medigraphic.com/pdfs/arcneu/ane-2016/ane163f.pdf

4. Yung Phang See, Whitehouse Kathrin, Lee Lucy, Khalil Hisham, McArdle Paul, C. Whitfield Peter. Management of CSF leak in base of skull fractures in adults, British Journal of Neurosurgery, 2016;30:6, 596-604, DOI: 10.1080/02688697.2016.1229746

5. González-Sánchez Jorge Glicerio, Chávez-Velázquez Ana Milagros, Velázquez-Santana Héctor. Manejo integral de fístulas de líquido cefalorraquídeo. An Orl Mex. 2012; 57:25-30.

6. Andrade-Lozano $P$, Salas-Galicia JE, Rodríguez-Briseño RA, Chávez-Méndez $M$, Gutiérrez-Vargas $R$, 
Garza-Talamas LM. Cirugía endoscópica transnasal de base de cráneo: algoritmo para el cierre de fístulas de líquido cefalorraquídeo. An Orl Mex. 2017; 62(1):1-10.

7. Tiffany Mathias BS, Levy J, Fatakia A, McCoul DE. Contemporary approach to the diagnosis and management of cerebrospinal fluid rhinorrhea. Ochsner J 2016;16: 136-142.

8. Felisati $G$, Bianchi A, Lozza $P$, Portaleone S. Italian multicentre study on intrathecal fluorescein for craniosinusal fistulae. Acta Otorhinolaryngologica Italica 2008;28: 159-163.

9. Silva LRF, Santos RP, Zymberg ST. Endoscopic endonasal approach for cerebrospinal fluid fistulae. Minimally Invasive Neurosurg 2006;49: 88-92.

10. Javadi SA, Samini $H$, Naderi $F$, Shirani $M$. The use of lowdose intrathecal fluorescein in endoscopic repair of cerebrospinal fluid rhinorrhea. Arch Iran Med 2013;16: 264-266.

11. Zhan R, Chen S, Xu S, Liu KJ, Li X. Postoperative lowflow cerebrospinal fluid leak of endoscopic endonasal transsphenoidal surgery for pituitary adenoma-wait and see, or lumbar drain? J Craniofac Surg 2015;26: 1261-1264.

12. Luis F. Castell. Manejo de fístulas de la lámela lateral de la lámina cribosa. Rev. Otorrinolaringol. Cir. Cabeza Cuello. 2007; 67: 46-52

13. Demarco RC, Tamashiro E, Valera FC, Anselmo-Lima WT. Use of a hypodense sodium fluorescein solution for the endoscopic repair of rhinogenic cerebrospinal fluid fistulae. Am J Rhinol 2007;21: 184-186. 